Pacific Journal of Mathematics

EXISTENCE AND GEOMETRY OF A FREE BOUNDARY
PROBLEM FOR THE HEAT EQUATION 


\title{
EXISTENCE AND GEOMETRY \\ OF A FREE BOUNDARY PROBLEM FOR THE HEAT EQUATION
}

\section{ANDREW ACKER AND KIRK LANCASTER}

\begin{abstract}
A periodic (in $t$ ) free boundary problem for the one-dimensional heat equation is examined. The existence and regularity of the (unique) solution is established and the geometry of the free boundary is shown to be no more complicated than the geometry of the fixed boundary.
\end{abstract}

0. Introduction. Free boundary problems (and moving boundary problems) arise in a large variety of contexts and have been studied for over one hundred years. There is an extensive literature on many aspects of free boundary problems including the existence, uniqueness, regularity, and stability of solutions and the qualitative properties of the free boundary ([14], [18]). Many applications, especially to continuum mechanics, have been considered since the work in the 1860's of Helmholtz and of Kirchhoff on fluid jets and of Neuman on the Stefan problem ([9], [10], [12], [15], [17], [27], [30]). In addition, the approximation of solutions and of free boundaries using numerical methods is well established (e.g. [16]).

Among parabolic problems, Stefan problems have generated a great deal of interest and an extensive literature (e.g. [11], [30]). We will examine a periodic free boundary problem for the one-dimensional heat equation which might be considered as a free boundary problem of Stefan type ([32]) in which the known (or "fixed") boundary varies periodically in time and the free boundary is determined by a prescribed flux condition (rather than a phase-change condition). In addition, this can be viewed as a model for certain processes involving chemical reactions. Alternatively, our problem might be viewed as a model problem in which techniques which have proven useful for certain elliptic free boundary problems (e.g. [1], [2], [5]) are applied to a particular parabolic problem.

We will use a trial-free-boundary approach based on an operator method to establish the existence of a solution to our free boundary problem. Trial-free-boundary methods have been used for over 70 years with success, as illustrated, for example, by the work of Cryer 
([16]). Our solution will be obtained as the limit of the fixed points of a sequence of contracting operators (similar to [2]) and the trialfree-boundary method based on this limiting process can be shown to converge (albeit slowly) to a solution as in [5].

An alternative approach for establishing the existence of a solution is to use a variational method. A natural functional to minimize in this case is the sum of the area and the heat flow (over one period). However, a minimizer of this functional does not lead to a solution of our problems. While another variational approach might be successful, we are unaware of an existence proof for our problem based on variational methods.

We will establish the regularity and, using (a variant of) the Lavrentiev principle, the uniqueness of the solution. We will then examine geometric properties of the free boundary. For certain harmonic or minimal surface free boundary problems, curves of constant gradient direction have been used to relate geometric properties of the free boundary to geometric properties of the fixed boundary (e.g. [4], [6]); these curves are related to the "nodal lines" of the Courant nodal line theorem ([13]) as well as to later work (e.g. [20], [25], [28]). We will use such curves to prove that the geometry of the free boundary is no more complicated than the geometry of the fixed boundary. To the best of our knowledge, the only previous application of this idea to parabolic free boundary problems is in the work of Friedman and Jensen ([19]).

1. Preliminaries. Given periodic functions $X^{*}(t)$ and $X(t)$ with period $\tau$ and, say, $X(t)>X^{*}(t)$ for each $t \in \mathfrak{R}$, let us denote

$$
\begin{aligned}
\Gamma^{*} & =\left\{\left(X^{*}(t), t\right): t \in \mathfrak{R}\right\}, \\
\Gamma & =\{(X(t), t): t \in \mathfrak{R}\}, \quad \text { and } \\
\Omega & =\left\{(x, t): X^{*}(t)<x<X(t), t \in \mathfrak{R}\right\} .
\end{aligned}
$$

Let us define $U=U\left(\Gamma^{*}, \Gamma\right) \in C^{2}(\Omega) \cap C^{0}(\bar{\Omega})$ to be the $\tau$-periodic (in $t$ ) solution of the Dirichlet problem

$$
\begin{array}{cc}
U_{t}=U_{x x} & \text { in } \Omega, \\
U\left(X^{*}(t), t\right)=1 & t \in \mathfrak{R}, \\
U(X(t), t)=0 & t \in \mathfrak{R} .
\end{array}
$$

We are interested in the following

Free Boundary Problem. Given $\Gamma^{*}$ as above, find $\Gamma$ (as above) such that if $U=U\left(\Gamma^{*}, \Gamma\right)$ then $U_{x} \in C^{0}(\Omega \cup \Gamma)$ and $U_{x}(X(t), t)=-1$. 
We will prove that if $X^{*}(t)$ is Lipschitz continuous, then the free boundary problem above has a solution $\Gamma=\{(X(t), t): t \in \mathfrak{R}\}$ with $X(t)$ Lipschitz continuous, this solution is unique, and the "geometry" of $\Gamma$ is no more complicated than that of $\Gamma^{*}$.

2. Existence. Suppose $X^{*}(t)$ is a $\tau$-periodic, Lipschitz continuous function with Lipschitz constant $\alpha$. Let $K=K(\alpha, \tau)$ denote the set of $\tau$-periodic, Lipschitz continuous functions $X(t)$ with Lipschitz constant $\alpha$ such that $X(t)>X^{*}(t)$ for $t \in \mathfrak{R}$.

Notation. At times, we will write $\Gamma \in K$ if $\Gamma=\Sigma(X)$ and $X \in K$, where

$$
\Sigma(X)=\{(X(t), t): t \in \mathfrak{R}\} .
$$

For $X_{1}, X_{2} \in K$ (resp. $\Gamma_{1}, \Gamma_{2} \in K$ with $\Gamma_{k}=\Sigma\left(X_{k}\right), k=1,2$ ), we define the form

$$
\left\|X_{1}-X_{2}\right\|=\left\|\Gamma_{1}-\Gamma_{2}\right\|=\max \left\{\left|X_{1}(t)-X_{2}(t)\right|: t \in \mathfrak{R}\right\} .
$$

For any $X \in K, \varepsilon \in(0,1)$, and $\Gamma=\Sigma(X)$, let

$$
\begin{aligned}
& \Phi_{\varepsilon}(\Gamma)=\{(x, t): U(x, t)=\varepsilon, t \in \mathfrak{R}\}, \\
& \Psi_{\varepsilon}(\Gamma)=\{(x+\varepsilon, t):(x, t) \in \Gamma\}, \quad \text { and } \\
& T_{\varepsilon}(\Gamma)=\Psi_{\varepsilon}\left(\Phi_{\varepsilon}(\Gamma)\right),
\end{aligned}
$$

where $U=U\left(\Gamma^{*}, \Gamma\right)$. We let $\phi_{\varepsilon}, \psi_{\varepsilon}$, and $t_{\varepsilon}$ be defined on $K$ so that $\Phi_{\varepsilon}(\Gamma)=\Sigma\left(\phi_{\varepsilon}(X)\right), \Psi_{\varepsilon}(\Gamma)=\Sigma\left(\psi_{\varepsilon}(X)\right)$, and $T_{\varepsilon}(\Gamma)=\Sigma\left(t_{\varepsilon}(X)\right)$, where $\Gamma=\Sigma(X)$ and $X \in K$. Notice that

$$
U\left(\phi_{\varepsilon}(X)(t), t\right)=\varepsilon,
$$

$\psi_{\varepsilon}(X)(t)=X(t)+\varepsilon$, and $t_{\varepsilon}(X)=\psi_{\varepsilon}\left(\phi_{\varepsilon}(X)\right)$ for $X \in K$.

LEMMA $1 . \phi_{\varepsilon}: K \rightarrow K$.

Proof. Let $X \in K$ and $\Gamma=\Sigma(X)$. Clearly $\phi_{\varepsilon}(X)$ is $\tau$-periodic, since $U$ is $\tau$-periodic in $t$, and $\phi_{\varepsilon}(X)(t)>X^{*}(t)$, since $X(t)>$ $X^{*}(t), U\left(X^{*}(t), t\right)=1$, and $U(X(t), t)=0$. Let $\left|\alpha_{0}\right| \geq \alpha, h>0$,

$$
\begin{gathered}
\Gamma_{h}=\Gamma+\left(\alpha_{0} h, h\right) \equiv\left\{\left(x+\alpha_{0} h, t+h\right):(x, t) \in \Gamma\right\} \\
\Gamma_{h}^{*}=\Gamma^{*}+\left(\alpha_{0} h, h\right), \quad \Omega_{h}=\Omega+\left(\alpha_{0} h, h\right), \quad \text { and } \\
U_{h}(x, t)=U\left(x-\alpha_{0} h, t-h\right)
\end{gathered}
$$


for $(x, t) \in \Omega_{h}$. Notice that $U_{h}(x, t) \geq U(x, t)$ in $\Omega \cap \Omega_{h}$, since $U_{h}=1 \geq U$ on $\Gamma_{h}^{*}, U_{h} \geq 0=U$ on $\Gamma$, and $U$ and $U_{h}$ both satisfy $u_{t}=u_{x x}$ in $\Omega_{h} \cap \Omega$. This means that $U$ is (weakly) decreasing on rays $x=x_{0}+\alpha_{0} t, x \geq x_{0}$ (and strictly decreasing if $\left|\alpha_{0}\right|>\alpha$ ). It follows that if $\left(x_{0}, t_{0}\right) \in \Phi_{\varepsilon}(\Gamma)$, then $\Phi_{\varepsilon}(\Gamma)$ lies to the left of the cone $\left\{\left(x+x_{0}, t+t_{0}\right):|t| \leq\left|\alpha_{0} x\right|, x>0\right\}$, since otherwise $U$ could not be monotonic on the ray $x=x_{0}+\alpha_{0}\left(t-t_{0}\right), x \geq x_{0}$. Thus $\phi_{\varepsilon}(x)$ is Lipschitz continuous with Lipschitz constant $\alpha$.

REMARK. If $\nabla U$ were continuous on $\bar{\Omega}$, we could simply have considered $\frac{\partial U}{\partial \nu}$ with $\nu=\left(\alpha_{0}, 1\right) /\left(\sqrt{\left.\alpha_{0}^{2}+1\right)}\right.$.

LEMMA 2. Suppose $\Gamma^{*}$ is a Lipschitz-continuously differentiable curve. Then $U_{x}<0$ on $\Gamma^{*}$.

Proof. This follows from the Hopf boundary point lemma for parabolic equations (e.g. [29], p. 170).

For two curves $\Gamma_{1}$ and $\Gamma_{2}$ with $\Gamma_{k}=\left\{\left(X_{k}(t), t\right): t \in \mathfrak{R}\right\}$, let us say $\Gamma_{1}<\Gamma_{2}$ if $X_{1}(t)<X_{2}(t)$ for all $t \in \mathfrak{R}$.

LemMA 3. There exist curves $\Gamma_{1}, \Gamma_{2} \in K$ with $\Gamma_{1}<\Gamma_{2}$ such that $T_{\varepsilon}\left(\Gamma_{1}\right)>\Gamma_{1}$ and $T_{\varepsilon}\left(\Gamma_{2}\right)<\Gamma_{2}$ for $\varepsilon \in(0,1)$ sufficiently small.

Proof. Choose $\Gamma_{1}=\Gamma^{*}+(\sigma, 0)=\left\{\left(x^{*}(t)+\sigma, t\right): t \in \mathfrak{R}\right\}$ with $\sigma>$ 0 small enough that $\left|\partial U_{1} / \partial x\right| \gg 1$ on $\Gamma_{1}$, where $U_{1}=U\left(\Gamma^{*}, \Gamma_{1}\right)$, and let $\Gamma_{2}=\left\{\left(x_{0}, t\right): t \in \mathfrak{R}\right\}$ for $x_{0}$ sufficiently large.

We define $\widetilde{K}=\widetilde{K}\left(\Gamma^{*}, \Gamma_{1}, \Gamma_{2}\right)$ by

$$
\widetilde{K}=\left\{X \in K: \Gamma_{1} \leq \Sigma(X) \leq \Gamma_{2}\right\}
$$

and write $\Gamma \in \widetilde{K}$ if $\Gamma=\Sigma(X)$ and $X \in \widetilde{K}$. Notice that if $\varepsilon \in(0,1)$ is sufficiently small, Lemma 3 implies that $T_{\varepsilon}: \widetilde{K} \rightarrow \widetilde{K}$. We may now state an existence theorem for fixed points of $T_{\varepsilon}$.

THEOREM 1. Let $\Gamma^{*}$ be a Lipschitz-continuously differentiable curve and suppose $U=U\left(\Gamma^{*}, \Gamma\right)$ is continuously differentiable in $\Omega \cup \Gamma^{*}$. Then, for $\varepsilon \in(0,1)$ sufficiently small, $t_{\varepsilon}: \widetilde{K} \rightarrow \widetilde{K}$ is a contraction. Thus, for each small $\varepsilon>0$, there exists a unique "fixed point" $\Gamma_{\varepsilon} \in \widetilde{K}$ of $T_{\varepsilon}$. 
Proof. Using Lemma 2 and the maximum principle, $\Phi_{\varepsilon}$ can be shown to be contracting as in [2]. Since $\Phi_{\varepsilon}$ is contracting, so is $T_{\varepsilon}$.

LEMMA 4 (Uniform Modulus of Continuity near $\Gamma$ ). Suppose $\Gamma \in \widetilde{K}$ and $V \in C^{2}(\Omega) \cap C^{0}(\bar{\Omega})$ is a solution of $V_{t}=V_{x x}$ in $\Omega=\Omega\left(\Gamma^{*}, \Gamma\right)$, $V=0$ on $\Gamma$, and $V=1$ on $\Gamma^{*}$. If $V$ is periodic in $t$ with period $\tau$, then

$$
V(x, y) \leq C \operatorname{dist}((x, t), \Gamma)
$$

for $(x, t) \in \Omega$, where $C=C\left(\Gamma^{*}, \Gamma_{1}, \Gamma_{2}, \alpha\right)>0$ is independent of $\Gamma$.

Proof. Let $\alpha>0$ denote the Lipschitz constant of $K$. Let $\left(X\left(t_{0}\right)\right.$, $\left.t_{0}\right) \in \Gamma$ and set

$$
\gamma=\left\{\left(X\left(t_{0}\right)-\alpha\left(t-t_{0}\right), t\right): t \in \mathfrak{R}\right\} .
$$

Let $\gamma^{*}=\gamma+\left(X^{*}\left(t_{0}\right)-X\left(t_{0}\right), 0\right)$ and let $\omega=\omega\left(t_{0}, \alpha\right)$ be the region between $\gamma^{*}$ and $\gamma$. Since the Lipschitz constant of $X^{*}(t)$ and $X(t)$ is (s) $\alpha, \Gamma \cap\left\{(x, t): t<t_{0}\right\}$ lies to the left of $\gamma$ and $\Gamma^{*} \cap\{(x, t): t<$ $\left.t_{0}\right\}$ lies to the left of $\gamma^{*}$. Let $w(x, t)$ be the solution of $w_{t}=w_{x x}$ in $\omega, w=0$ on $\gamma$, and $w=1$ on $\gamma^{*}$. Using the fact that $0<V<1$ in $\Omega$ and $0<w<1$ in $\omega$, it is easily seen that $V \leq w$ on the parabolic boundary of the region $\Omega \cap \omega \cap\left\{(x, t): t \leq t_{0}\right\}$. Therefore, the maximum principle implies that $V \leq w$ in $\Omega \cap \omega \cap\left\{(x, t): t \leq t_{0}\right\}$. Now

$$
w(x, t)=\phi(x+\alpha t)
$$

for some $\phi \in C^{2}\left(\left[X^{*}\left(t_{0}\right)+\alpha t_{0}, X\left(t_{0}\right)+\alpha t_{0}\right]\right)$. Then

$$
\phi^{\prime \prime}(s)=\alpha \phi^{\prime}(s)
$$

and so $\phi(s)=A e^{\alpha s}+B$. Since $\phi\left(X^{*}\left(t_{0}\right)+\alpha t_{0}\right)=1$ and $\phi\left(X\left(t_{0}\right)+\alpha t_{0}\right)$ $=0$, we see that $\phi(s)=\left(e^{\alpha s_{1}}-e^{\alpha s}\right) /\left(e^{\alpha s_{1}}-e^{\alpha s_{0}}\right)$, where $s_{0}=X^{*}\left(t_{0}\right)+$ $\alpha t_{0}$ and $s_{1}=X\left(t_{0}\right)+\alpha t_{0}$. Thus

$$
w(x, t)=\frac{e^{\alpha\left(x_{1}+\alpha t_{0}\right)}-e^{\alpha(x+\alpha t)}}{e^{\alpha\left(x_{1}+\alpha t_{0}\right)}-e^{\alpha\left(x_{0}+\alpha t_{0}\right)}},
$$

where $x_{0}=X^{*}\left(t_{0}\right)$ and $x_{1}=X\left(t_{0}\right)$, and, in particular,

$$
w\left(x, t_{0}\right)=\frac{e^{\alpha x_{1}}-e^{\alpha x}}{e^{\alpha x_{1}}-e^{\alpha x_{0}}}
$$


for $x \in\left[x_{0}, x_{1}\right]$. Then $V\left(x_{1}-h, t_{0}\right) \leq w\left(x_{1}-h, t_{0}\right)$ and

$$
\begin{aligned}
w\left(x_{1}-h, t_{0}\right) & =\frac{e^{\alpha x_{1}}}{e^{\alpha x_{1}}-e^{\alpha x_{0}}}\left(1-e^{-\alpha h}\right) \\
& =\frac{e^{\alpha x_{1}}}{e^{\alpha x_{1}}-e^{\alpha x_{0}}} \alpha h+O\left((\alpha h)^{2}\right) \\
& \leq C_{1} h
\end{aligned}
$$

for $h>0$ sufficiently small and $C_{1}=e^{\alpha x_{1}} /\left(e^{\alpha x_{1}}-e^{\alpha x_{0}}\right)+1$, for example. Now

$$
\operatorname{dist}\left(\left(x, t_{0}\right), \Gamma\right) \geq \operatorname{dist}\left(\left(x, t_{0}\right), \gamma\right)
$$

and

so

$$
\operatorname{dist}\left(\left(x_{1}-h, t_{0}\right), \gamma\right)=\frac{1}{\sqrt{1+\alpha^{2}}} h,
$$

$$
V\left(x-h, t_{0}\right) \leq C \operatorname{dist}\left(\left(x, t_{0}\right), \Gamma\right),
$$

where $C=\left(1+\alpha^{2}\right)^{-1 / 2} C_{1}$. Notice that $x_{1} \geq x_{0}+\sigma$ (recall $\Gamma_{1}$ $\left.=\Gamma^{*}+(\sigma, 0)\right)$ and so $C_{1}$ has a finite upper bound independent of $\Gamma$.

Construction of a candidate for a solution. For some $\varepsilon_{0}>0, T_{\varepsilon}$ has a "fixed point" $\Gamma_{\varepsilon} \in \widetilde{K}$ for all $0<\varepsilon \leq \varepsilon_{0}$ provided $\Gamma^{*}$ is sufficiently regular (Theorem 1). Since each $\Gamma \in \widetilde{K}$ satisfies $\Gamma_{1} \leq \Gamma \leq \Gamma_{2}$ and $X_{1}(t) \leq X(t) \leq X_{2}(t), t \in \Re$, where $\Gamma=\Sigma(X)$, the ArzelàAscoli Theorem implies that there exists a sequence $\left\{\varepsilon_{n}\right\}$ converging to 0 and $X^{0} \in \widetilde{K}$ such that $X_{\varepsilon_{n}} \rightarrow X^{0}$ in $C^{0}(\Re)$ and $\Gamma_{n} \rightarrow \Gamma^{0}$ uniformly as $n \rightarrow \infty$, where $\Gamma^{0}=\Sigma\left(X^{0}\right)$ and $\Gamma_{n}=\Sigma\left(X_{\varepsilon_{n}}\right)$. Let us denote $U\left(\Gamma^{*}, \Gamma^{0}\right)$ by $U^{0}, \Omega\left(\Gamma^{*}, \Gamma^{0}\right)$ by $\Omega^{0}, U\left(\Gamma^{*}, \Gamma_{n}\right)$ by $U_{n}$, and $\Omega\left(\Gamma, \Gamma_{n}\right)$ by $\Omega_{n}$.

THEOREM 2. Suppose $\Gamma^{*}$ is sufficiently regular that the hypotheses of Theorem 1 hold. Then $U_{x}^{0} \in C^{0}\left(\bar{\Omega}^{0}\right)$ and $U_{x}^{0}=-1$ on $\Gamma^{0}$. In particular, $\Gamma^{0}$ solves the free boundary problem. Further, this solution is unique.

Proof. Notice that $\left\|\Gamma_{n}-\Gamma^{0}\right\|_{\infty} \equiv \max \left\{\left|X_{\varepsilon_{n}}(t)-X^{0}(t)\right|: t \in \mathfrak{R}\right\}$ goes to zero as $n \rightarrow \infty$. Using Lemma 4 and the fact that $U_{n}=U^{0}=1$ on $\Gamma^{*}$, we see that $U_{n} \rightarrow U^{0}$ uniformly on compact subsets of $\Omega^{0} \cap \Gamma^{*}$. Let

$$
V_{n}(x, t)=\frac{U_{n}(x, t)-U_{n}\left(x-\varepsilon_{n}, t\right)}{\varepsilon_{n}}
$$


in $\widehat{\Omega}_{n}=\Omega\left(\Gamma^{*}+\left(\varepsilon_{n}, 0\right), \Gamma_{n}\right)$. Then $V_{n}=\left(0-\varepsilon_{n}\right) / \varepsilon_{n}=-1$ on $\Gamma_{n}$, since $T_{\varepsilon}\left(\Gamma_{\varepsilon}\right)=\Gamma_{\varepsilon}$ and so $\phi_{\varepsilon}\left(X_{\varepsilon}\right)(t)=X_{\varepsilon}(t)-\varepsilon$. Also notice $\left(V_{n}\right)_{t}=\left(V_{n}\right)_{x x}$ in $\widehat{\Omega}_{n}$ and, from the mean-value theorem,

$$
V_{n}(x, t)=\frac{\partial}{\partial x}\left(U_{n}\left(\lambda_{n}(t), t\right)\right)
$$

for some $\lambda_{n}(t) \in\left(x-\varepsilon_{n}, x\right)$. Now suppose $(x, t) \in \Omega^{0}$ and $n$ is large enough that $(x, t) \in \widehat{\Omega}_{n}$. Since, as $n \rightarrow \infty, U_{n}$ converges uniformly on compact subsets of $\Omega^{0}$ to $U^{0}$, we see that

$$
\frac{\partial}{\partial x}\left(U_{n}\left(\lambda_{n}(t), t\right)\right) \rightarrow \frac{\partial}{\partial x}\left(U^{0}(x, t)\right) \quad \text { as } n \rightarrow \infty
$$

(e.g. [29]) and hence

$$
V_{n}(x, t) \rightarrow \frac{\partial}{\partial x}\left(U^{0}(x, t)\right)
$$

as $n \rightarrow \infty$.

Let $\Omega_{p}^{0}=\left\{(x, t) \in \Omega: U^{0}(x, t)<\frac{1}{2}\right\}$ and let $\Gamma_{p}^{*}$ denote the left boundary of $\Omega_{p}^{0}$. Let $V \in C^{2}\left(\Omega_{p}^{0}\right) \cap C^{0}\left(\bar{\Omega}_{p}^{0}\right)$ satisfy $V_{t}=V_{x x}$ in $\Omega_{p}^{0}$, $V=-1$ on $\Gamma^{0}, V=U_{x}$ on $\Gamma_{p}^{*}$, and $V(x, t)$ is $\tau$-periodic in $t$. Since $V_{n}=-1$ on $\Gamma_{n}$, a result analogous to Lemma 4 implies

$$
\left|V(x, t)-V_{n}(x, t)\right| \leq k_{n}
$$

for $(x, t)$ an element of the right boundary of $\Omega^{0} \cap \Omega_{n}$, where $k_{n} \rightarrow 0$ as $n \rightarrow \infty$. Since $V(x, t)=U_{x}^{0}(x, t)$ and $V_{n}(x, t) \rightarrow U_{x}^{0}(x, t)$ as $n \rightarrow \infty$, for $(x, t) \in \Gamma_{p}^{*}$, we see that $V_{n}(x, t) \rightarrow V(x, t)$ as $n \rightarrow \infty$, for $(x, t) \in \Gamma_{p}^{*}$. Thus

$$
V_{n} \rightarrow V
$$

uniformly on compacta in $\Omega_{p}^{0}$ and so $V=U_{x}^{0}$. Since $V(x, t) \rightarrow-1$ as $(x, t) \in \Omega^{0}$ approaches $\Gamma^{0}, U_{x}=-1$ on $\Gamma^{0}$.

Turning to the uniqueness question, we will show, using an adaptation of the Lavrentiev principle ([23], [24]) that the existence of two distinct solutions $\Gamma^{0}, \Gamma^{1} \in K$ leads to a contradiction. Assuming $\Gamma^{0} \geq \Gamma^{1}$ is false, let $\sigma>0$ be the least number such that $\Gamma^{1} \leq \Gamma^{2} \equiv \Gamma^{0}+(\sigma, 0)$. Then two applications of the maximum principle show that

$$
U\left(\Gamma^{*}, \Gamma^{1}\right)<U\left(\Gamma^{*}, \Gamma^{2}\right)<U\left(\Gamma^{*}+(\sigma, 0), \Gamma^{0}+(\sigma, 0)\right)
$$

throughout $\Omega\left(\Gamma^{*}+(\sigma, 0), \Gamma^{1}\right)$, where the inequalities reduce to equality at any point $p_{0} \in \Gamma^{1} \cap \Gamma^{2}$. Using the regularity results of $\S 3$ (which 
apply to both $\Gamma^{0}$ and $\Gamma^{1}$ ) and the Hopf boundary point lemma, we see that

$$
\begin{aligned}
-1 & =U_{x}\left(\Gamma^{*}, \Gamma^{1}\right)\left(p_{0}\right)>U_{x}\left(\Gamma^{*}+(\sigma, 0), \Gamma+(\sigma, 0)\right)\left(p_{0}\right) \\
& =U_{x}\left(\Gamma^{*}, \Gamma^{0}\right)\left(p_{0}-(\sigma, 0)\right)=-1 .
\end{aligned}
$$

From now on, we will write $\Gamma^{0}$ as $\Gamma$.

3. Regularity of the free boundary. In this section, we will let $\Gamma^{*}=$ $\left\{\left(X^{*}(t), t\right): t \in \mathfrak{R}\right\}$, where $X^{*}$ is a $\tau$-periodic, Lipschitz continuous function with Lipschitz constant $\alpha$ and we will let $\Gamma \in K$ be the solution of the free boundary problem, whose existence follows from Theorem 2. We will let $U=U\left(\Gamma^{*}, \Gamma\right)$ and $\Omega=\Omega\left(\Gamma^{*}, \Gamma\right)$.

LEMMA 5. The level curves of $U$ are Lipschitz continuous with Lipschitz constant $\alpha$.

Proof. This follows from Lemma 1.

LEMMA 6. $U_{t}$ and $U_{x x}$ are uniformly bounded in $\Omega$.

Proof. Recall $U\left(X_{\varepsilon}(t), t\right)=\varepsilon$ for all $0<\varepsilon \leq \varepsilon_{0}$. If we differentiate with respect to $t$, we obtain

$$
U_{x}\left(X_{\varepsilon}(t), t\right) X_{\varepsilon}^{\prime}(t)+U_{t}\left(X_{\varepsilon}(t), t\right)=0
$$

and so

$$
\left|U_{t}\left(X_{\varepsilon}(t), t\right)\right|=\left|X_{\varepsilon}^{\prime}(t)\right|\left|U_{x}\left(X_{\varepsilon}(t), t\right)\right| \leq \alpha\left|U_{x}\left(X_{\varepsilon}(t), t\right)\right| .
$$

Now $U_{x}(x, t) \rightarrow-1$ as $(x, t) \rightarrow \Gamma$ and $U_{x}$ is bounded in $\bar{\Omega}$, so $\left|U_{t}\right|$ is bounded in $\Omega$. Since $U_{x x}=U_{t}, U_{x x}$ is also bounded. $\left.\frac{1}{2}\right\}$.

LEMMA 7. $U_{x t}$ is uniformly bounded in $\Omega_{p}=\{(x, t): U(x, t)<$

Proof. We will assume that $\Gamma^{*}$ is as regular as we wish, and prove the lemma in $\Omega$, since otherwise we could replace $U$ by $V(x, t)=$ $2 U(x, t)$ and $\Omega$ by $\Omega_{p}$ and notice that the left boundary of $\Omega_{p}$ is smooth. Let us define

$$
V_{h}(x, t)=U_{x}(x, t+h)-U_{x}(x, t)
$$

in $\Omega_{h}=\Omega \cap(\Omega-(0, h))$. Notice that $\Omega_{h} \rightarrow \Omega$ as $h \rightarrow 0+$. Let $\Gamma_{h}=\Sigma\left(X_{h}\right)$ and $\Gamma_{h}^{*}=\Sigma\left(X_{h}^{*}\right)$, where $X_{h}(t)=\min \{X(t), X(t+h)\}$ and $X_{h}^{*}(t)=\max \left\{X^{*}(t), X^{*}(t+h)\right\}$. Then

$$
\left|V_{h}\left(X_{h}(t), t\right)\right|=\left|U_{x}\left(X_{h}(t), t+h\right)-U_{x}\left(X_{h}(t), t\right)\right| .
$$


Suppose, say, that $X(t+h) \geq X(t)$ and so $X_{h}(t)=X(t)$. Then

$$
\begin{aligned}
\left|V_{h}\left(X_{h}(t), t\right)\right| & =\left|U_{x}(X(t), t+h)-U_{x}(X(t), t)\right| \\
& =\left|U_{x}(X(t), t+h)+1\right| \\
& =\left|U_{x}(X(t), t+h)-U_{x}(X(t+h), t+h)\right| \\
& =\left|U_{x x}(\lambda(t), t+h)\right||X(t+h)-X(t)| \\
& \leq M \alpha|h|
\end{aligned}
$$

where $\left|U_{x x}\right| \leq M$ in $\Omega$ and $\lambda(t) \in(X(t), X(t+h))$. If $X(t+h)<$ $X(t)$, a similar argument yields the same estimate. Now $U$ is smooth in $\Omega \cup \Gamma^{*}$ (by our assumption of regularity of $\Gamma^{*}$ ) and so there exists $M_{1}$ such that $\left|U_{x t}\right| \leq M_{1}$ on $\left\{(x, t): \frac{1}{6}<U(x, t)<1\right\}$. Since $V_{h}(x, t) / h$ is the difference quotient for $U_{x t}(x, t)$, we see that for $M_{2}>M_{1}$ and $h>0$ sufficiently small,

$$
\left|V_{h}\left(X_{h}^{*}(t), t\right)\right| \leq M_{2}|h| \text {. }
$$

Since $\left(V_{h}\right)_{t}=\left(V_{h}\right)_{x x}$ in $\Omega_{h}$ and $\left|V_{h}(x, t)\right| \leq M_{3}|h|$ on $\partial\left(\Omega_{h}\right)$, where $M_{3}=\max \left\{M \alpha, M_{2}\right\}$, we obtain

$$
\left|\frac{U_{x}(x, t+h)-U_{x}(x, t)}{h}\right| \leq M_{3} \text { in } \Omega_{h}
$$

for any $h$ small enough. It follows that $\left|U_{x t}\right| \leq M_{3}$ in $\Omega$.

LEMMA 8. $U_{t}$ is continuous on $\Omega \cup \Gamma$.

Proof. Let us begin by defining $U_{t}$ on $\Gamma$. Set $f_{\varepsilon}=U_{t}(X(t)-\varepsilon, t)$ for $\varepsilon>0$ small. Notice that $f_{\varepsilon} \in C^{0}(\mathfrak{R})$ and

$$
\begin{aligned}
\left|f_{\varepsilon}(t)-f_{\beta}(t)\right| & =\left|U_{t}(X(t)-\varepsilon, t)-U_{t}(X(t)-\beta, t)\right| \\
& \leq\left|U_{x t}\left(\lambda_{\varepsilon, \beta}(t)\right)\right||\varepsilon-\beta| \leq M_{3}|\varepsilon-\beta|,
\end{aligned}
$$

where $X(t)-\varepsilon<\lambda_{\varepsilon, \beta}(t)<X(t)-\beta$ if $\varepsilon>\beta$. Then $f_{\varepsilon}(t)$ converges uniformly as $\varepsilon \rightarrow 0+$ to a function $f \in C^{0}(\mathfrak{R})$. Let us define $U_{t}(X(t), t)=f(t)$.

Now we wish to show that $U_{t} \in C^{0}(\Omega \cup \Gamma)$. Let $\left(x_{0}, t_{0}\right)=$ $\left(X\left(t_{0}\right), t_{0}\right) \in \Gamma$ and let $(x, t) \in \Omega$. Then

$$
\begin{aligned}
\mid U_{t}(x & , t)-U_{t}\left(x_{0}, t_{0}\right) \mid \\
& \leq\left|U_{t}\left(x_{0}, t_{0}\right)-U_{t}(X(t), t)\right|+\left|U_{t}(X(t), t)-U_{t}(x, t)\right| \\
& \leq\left|f\left(t_{0}\right)-f(t)\right|+M_{3}|x-X(t)| \\
& \leq \xi\left(t-t_{0}\right)+M_{3}\left(\left|x-x_{0}\right|+\left|x_{0}-X(t)\right|\right) \\
& \leq \xi\left(t-t_{0}\right)+M_{3}\left|x-x_{0}\right|+M_{3} \alpha\left|t-t_{0}\right| \rightarrow 0
\end{aligned}
$$

as $t \rightarrow t_{0}, x \rightarrow x_{0}$, where $\xi(\tau) \rightarrow 0$ as $\tau \rightarrow 0$. 
LeMMA 9. $\Gamma$ is $C^{1}$ (i.e. $X \in C^{1}(\Re)$ ).

Proof. Since $U\left(X_{\varepsilon}(t), t\right)=\varepsilon, X_{\varepsilon}^{\prime}(t)=-U_{t}\left(X_{\varepsilon}(t), t\right) / U_{x}\left(X_{\varepsilon}(t), t\right)$. Since $U_{x} \in C^{0}(\Omega \cup \Gamma), U_{x}=-1$ on $\Gamma$, and $U_{t}$ is bounded and continuous, we have

$$
X_{\varepsilon}^{\prime} \rightarrow-U_{t}(X(t), t)
$$

uniformly in $t$ as $\varepsilon \rightarrow 0+$. Now

$$
\begin{aligned}
X(t+h)-X(t) & =\lim _{\varepsilon \rightarrow 0}\left(X_{\varepsilon}(t+h)-X_{\varepsilon}(t)\right) \\
& =\lim _{\varepsilon \rightarrow 0+} \int_{t}^{t+h} X_{\varepsilon}^{\prime}(s) d s=-\int_{t}^{t+h} U_{t}(X(s), s) d s
\end{aligned}
$$

and so

$$
X^{\prime}(t)=\lim _{h \rightarrow 0}-\frac{1}{h} \int_{t}^{t+h} U_{t}(X(s), s) d s=-U_{t}(X(t), t) .
$$

Since $U_{t} \in C^{0}(\Omega \cup \Gamma), X^{\prime} \in C^{0}(\mathfrak{R})$.

TheOREM 3. $\Gamma$ is $C^{\infty}$ and $U \in C^{\infty}(\Omega \cup \Gamma)$.

Proof. This follows from Lemma 9 and [22].

4. Geometry of the free boundary. As in the previous section, we let $\Gamma^{*}=\left\{\left(x^{*}(t), t\right): t \in \mathfrak{R}\right\}$ and $\Gamma=\{(X(t), t): t \in \mathfrak{R}\}$ be the solution of the free boundary problem, $U=U\left(\Gamma^{*}, \Gamma\right)$, and $\Omega=\Omega\left(\Gamma^{*}, \Gamma\right)$. We will compare the geometry of $\Gamma$ to that of $\Gamma^{*}$.

Let $\nu=a \vec{i}+b \vec{j}$ with $a>0$ and suppose that $p_{0}=\left(x_{0}, t_{0}\right)=$ $\left(X\left(t_{0}\right), t_{0}\right) \in \Gamma$ is a $\nu$-minimum of $\Gamma$ (i.e. $h(t)=(X(t), t) \cdot \nu$ has a strict local minimum at $t_{0}$ or, for some $\delta>0$, if $\left|t-t_{0}\right|<\delta$, then $h(t) \geq h\left(t_{0}\right)$ and for some $t_{1}, t_{2} \in\left(t_{0}-\delta, t_{0}+\delta\right)$ with $t_{1}<t_{0}<t_{2}$, $h\left(t_{1}\right)>h\left(t_{0}\right)$, and $\left.h\left(t_{2}\right)>h\left(t_{0}\right)\right)$. Then $U_{\lambda}\left(p_{0}\right)=0$ if $\lambda=-b \vec{i}+a \vec{j}$. Also $U_{\lambda}(X(t), t)>0$ if $t>t_{0}$ is near $t_{0}$ and $U_{\lambda} \leq 0$ if $t<t_{0}$ is near $t_{0}$. Here $U_{\lambda}(x, t)=\nabla U \cdot \lambda=-b U_{x}(x, t)+a U_{t}(x, t)$.

LEMMA 10. For any direction $\lambda$ and any compact $M \subset \Omega$, there are only a finite number of points in $M$ at which $U_{\lambda}=0$ and $\left(U_{\lambda}\right)_{x}=0$ simultaneously.

Proof. Let $\phi(x, t)=U_{\lambda}(x, t)$. Then $\phi_{t}=\phi_{x x}$ in $\Omega$. Now let $p_{0}=\left(x_{0}, t_{0}\right) \in M$. Suppose first that $\phi\left(p_{0}\right) \neq 0$ or $\phi_{x}\left(p_{0}\right) \neq 0$. Then we can find a (closed) rectangle $N$ centered at $p_{0}$ such that either $\phi(x, t) \neq 0$ or $\phi_{x}(x, t) \neq 0$ for each $(x, t) \in N$. Suppose now that 
$\phi\left(p_{0}\right)=\phi_{x}\left(p_{0}\right)=0$. Let $N$ be the rectangle given in Theorem B of [8], where $\delta, \varepsilon>0$. Let $z(t)$ denote the number of zeros of $\phi(\cdot, t)$ in the interval $I=\left[x_{0}-\varepsilon, x_{0}+\varepsilon\right]$ and let $k(t)$ denote the number of common zeros of $\phi(\cdot, t)$ and $\phi_{x}(\cdot, t)$ in $I$, for $t \in J=\left[t_{0}-\delta, t_{0}+\delta\right]$. Using [8], we see that $z(t)$ is nonincreasing and $z\left(t_{1}\right) \geq z\left(t_{2}\right)+k\left(t_{2}\right)$ when $t_{1}, t_{2} \in J$ with $t_{1}<t_{2}$. Since $z\left(t_{0}-\delta\right)$ is finite, a simple counting argument, together with the maximum principle, shows that $S=\sum_{t \in J} k(t)<\infty$; in fact, $S \leq \frac{1}{2} z\left(t_{0}-\delta\right)$. Hence, the number of points in $N$ at which $\phi=\phi_{x}=0$ is finite. Since $M$ can be covered by a finite number of rectangles of the two types above, which have either none or a finite number of points at which $\phi=\phi_{x}=0$, we are done.

LEMMA 11. Let $p_{0} \in \Omega$ be a point of the set $\sigma=\{(x, t) \in \Omega$ : $\left.U_{\lambda}(x, t)=0\right\}$ at which $\nabla U_{\lambda} \neq \overrightarrow{0}$. Then $\sigma$ is (or can be extended to be) a smooth (i.e. $C^{\infty}$ ) arc near $p_{0}$.

Proof. The proof follows from the implicit function theorem.

LEMMA 12. Let $\sigma$ be any directed arc such that $U_{\lambda}=0$ on $\sigma$ and $U_{\lambda}>0$ locally to the right of $\sigma$. Then the map $f(p)=-b U(p)+$ $a U_{x}(p)$ is strictly increasing on $\sigma$.

Proof. Let $\sigma$ be parametrized by $p(s)=(x(s), t(s))$ with $\left|p^{\prime}(s)\right| \neq$ 0 (except possibly at a finite number of points). Let $s_{1} \in \mathfrak{R}$ and $\left(x_{1}, t_{1}\right)=p\left(s_{1}\right) \in \gamma$. Let us set $F(s)=f(x(s), t(s))$. Then $F^{\prime}(s)=$ $\left(-b U_{t}(p(s))+a U_{x t}(p(s))\right) t^{\prime}(s)$. If $t^{\prime}\left(s_{1}\right) \geq 0$, then $\left(-b U_{x}+a U_{t}\right)_{x}$ $\geq 0$ and so $-b U_{t}+a U_{x t}=-b U_{x x}+a U_{x t} \geq 0$. If $t^{\prime}\left(s_{1}\right)<0$, then $\left(-b U_{x}+a U_{t}\right)_{x} \leq 0$ and so $-b U_{t}+a U_{x t} \leq 0$. In either case, $F^{\prime}\left(s_{1}\right) \geq$ 0 . Since $s_{1}$ was arbitrary, we see that $F(s)$ is (weakly) increasing. To see that $F(s)$ is strictly increasing, suppose $F^{\prime}(s)=0$ for $s$ in an open interval $I$. There are only a finite number of points in any compact subset of $\Omega$ at which $U_{\lambda}=\left(U_{\lambda}\right)_{x}=0$. We may thus assume that $U_{\lambda} \neq 0$ on $p(I)$; this implies $t^{\prime} \equiv 0$ on $I$. Then $\sigma$ is horizontal (near $p(I))$ and so $\left(U_{\lambda}\right)_{x} \equiv 0$ on $p(I)$, a contradiction.

Let us now define a directed arc $\gamma=\gamma\left(\nu, p_{0}\right)$ from $p_{0}$ into $\Omega$ such that $U_{\lambda}=0$ on $\gamma$ and $U_{\alpha}>0$ locally to the right of $\gamma$. Let $D=\left\{(x, t) \in \Omega: U_{\lambda}(x, t)>0\right\}$ and let $D^{0}$ be the component of $D$ whose boundary contains the upper portion (i.e. $t>t_{0}$ ) of some neighborhood of $p_{0}$ in $\Gamma$. Let $\gamma\left(\nu, p_{0}\right)$ be the component of $\partial D^{0} \cap\left(\Omega \cup\left\{(x, t) \in \Gamma: U_{\lambda}(x, t)=0\right\}\right)$ which contains $p_{0}$. We give 
$\gamma=\gamma\left(\nu, p_{0}\right)$ the direction which keeps $D^{0}$ locally to the right of $\gamma$ at every point of $\gamma \cap \Omega$. In a neighborhood of each point $p \in \gamma$ with $\nabla U_{\lambda}(p) \neq \overrightarrow{0}, \gamma$ is a smooth arc with $U_{\lambda}<0$ to the left of $\gamma$.

LEMMA 13. The set $\gamma=\gamma\left(\nu, p_{0}\right)$ is a Jordan arc.

Proof. Since $\gamma$ cannot intersect itself (Lemma 12 or the maximum principle, since $U_{\lambda}$ cannot vanish identically on an open subset of $\Omega$ ), the only possible difficulty would have to occur at a point $p_{3}=$ $\left(x_{3}, t_{3}\right) \in \gamma$ with $\nabla U_{\lambda}\left(p_{3}\right)=\overrightarrow{0}$. By Theorem B of [8] and Lemma 10 , we may assume that in the rectangle $N=\left[x_{3}-\varepsilon, x_{3}+\varepsilon\right] \times$ $\left[t_{3}-\delta, t_{3}+\delta\right]$ (for some $\left.\varepsilon, \delta>0\right), U_{\lambda}\left(\cdot, t_{3}+\delta\right.$ ) has at most one zero in $\left[x_{3}-\varepsilon, x_{3}+\varepsilon\right], U_{\lambda}\left(\cdot, t_{3}-\delta\right)$ has at least two zeros in $\left[x_{3}-\right.$ $\left.\varepsilon, x_{3}+\varepsilon\right]$, and $U_{\lambda x}(p) \neq 0$ if $p_{3} \neq p \in N \cap \gamma$. Either $U_{\lambda}\left(\cdot, t_{3}\right)$ changes signs at $x_{3}$ or it does not. First if, say, $U_{\lambda}\left(x, t_{3}\right)>0$ for $x \neq x_{3}$ with $\left(x, t_{3}\right) \in N$, then $U_{\lambda}(x, t)>0$ if $(x, t) \in N$ with $t>t_{3}$ and there exist (unique) $x_{L}, x_{R} \in C^{0}\left(\left[t_{3}-\delta, t_{3}\right]\right)$ such that $x_{L}\left(t_{3}\right)=x_{R}\left(t_{3}\right)=x_{3}, x_{3}-\varepsilon \leq x_{L}(t)<x_{R}(t) \leq x_{3}+\varepsilon$ for all $t \in$ $\left[t_{3}-\delta, t_{3}\right), U_{\lambda}\left(x_{L}(t), t\right)=U_{\lambda}\left(x_{R}(t), t\right)=0$ for $t \in\left[t_{3}-\delta, t_{3}\right]$, and $U_{\lambda}(x, t)>0$ if $(x, t) \in N, t<t_{3}$, and $x \notin\left[x_{L}(t), x_{R}(t)\right]$. Then $\gamma \cap N=\left\{\left(x_{R}(t), t\right)\right\} \cup\left\{\left(x_{L}(t), t\right)\right\}$. Second, if $U_{\lambda}\left(x, t_{3}\right)<0$ when $x_{3}-\varepsilon<x<x_{3}$ and $U_{\lambda}\left(x, t_{3}\right)>0$ when $x_{3}<x<x_{3}+\varepsilon$, for example, then there exists $x_{R} \in C^{0}\left(\left[t_{3}-\delta, t_{3}+\delta\right]\right)$ such that $U_{\lambda}\left(x_{R}(t), t\right)=0$ for $t \in\left[t_{3}-\delta, t_{3}+\delta\right]$ and $U_{\lambda}(x, t)<0$ if $(x, t) \in N$ and $x>x_{R}(t)$. Then $\gamma \cap N=\left\{\left(x_{R}(t), t\right)\right\}$. In each case, $\gamma$ is a Jordan arc in a neighborhood of $p_{3}$.

REMARK. (a) $\gamma$ is double-point free.

(b) If $\gamma$ enters $\Omega$ at a point $p_{0} \in \Gamma$, then $\gamma$ can never return to $\Gamma$, since $f(p)=-a$ on $\Gamma$.

LEMMA 14. Let $\gamma$ be as above. Then

(a) $\gamma$ cannot have a strict local $(0,1)$-minimum in $\Omega$.

(b) $\gamma$ cannot have more than one strict local $(0,1)$-maximum in $\Omega$.

Proof. Notice that $\phi \equiv-b U_{x}+a U_{t}$ satisfies $\phi_{t}=\phi_{x x}$ in $\Omega$ and $\phi=0$ on $\gamma$. Suppose $\gamma$ has a strict local $(0,1)$-minimum at $\left(x_{0}, t_{0}\right)$. Then, for some sufficiently small $\varepsilon>0$, there is a connected, simply connected region $\omega$ bounded below by an arc of $\gamma$ and above by an interval of $t=t_{0}+\varepsilon$ such that $\left(x_{0}, t_{0}\right) \in \partial \omega$. Since $\phi=0$ on $\partial \omega \cap \gamma$, which is the parabolic boundary of $\omega$, the maximum 
principle implies that $\phi \equiv 0$ and so $\phi \equiv 0$ in $\Omega$, a contradiction. Conclusion (b) follows immediately from (a).

COROllary. Let $(x(s), t(s))$ be a parametrization of $\gamma$. Then $t(s)$ is monotonic or there exists $s_{0}$ such that $t(s)$ is increasing on $s \leq s_{0}$ and decreasing on $s \geq s_{0}$.

LEMMA 15. Suppose $\Gamma^{*}$ is not a vertical line, $p_{0}=\left(x_{0}, t_{0}\right) \in \Gamma$ is $a \nu$-minimum of $\Gamma$, where $\nu=a \vec{i}+b \vec{j}$, and $\gamma=\gamma\left(\nu, p_{0}\right)$ begins at $p_{0}$. Then, for some $k>0, \gamma$ lies between $t=t_{0}+k$ and $t=t_{0}-k$.

Proof. Let $(x(s), t(s))$ be an arclength parametrization of $\gamma$ and suppose $t(s) \rightarrow \infty$ as $s \rightarrow \infty$. From the corollary above, we see that $t(s)$ is an increasing function and $\gamma$ can be represented as $x=\psi(t)$, $t \geq t_{0}$. For each natural number $n$, let

$$
\gamma_{n}=\{(x, t-n \tau):(x, t) \in \gamma\}
$$

and notice that $\gamma_{n}$ is represented by

$$
x=\psi_{n}(t) \equiv \psi(t+n \tau), \quad t \geq t_{0}-n \tau .
$$

Now the $\gamma_{n}$ do not intersect, since if $(x, t) \in \gamma_{n} \cap \gamma_{m}$, then $(x, t+n \tau)$ and $(x, t+m \tau)$ are in $\gamma$ and Lemma 12 implies $m=n$. This implies $m=n$. This implies $\psi_{n+1}(t)<\psi_{n}(t)$ in the domain $t \geq t_{0}$. For any fixed $t \geq t_{0},\left(\psi_{n}(t)\right)$ is a decreasing sequence bounded below by $X^{*}(t)$ (since $\gamma_{n} \subset \Omega$ for each $n$ ) and so $\tilde{\psi}(t)=\lim _{n \rightarrow \infty} \psi_{n}(t)$ exists. Now $\tilde{\psi}(t)=X^{*}(t)$ for all $t \geq t_{0}$ by Theorem $A$ of [8] and so $\gamma_{n} \rightarrow \Gamma^{*}$ as $n \rightarrow \infty$. Since $-b U+a U_{x}$ is increasing on $\gamma_{n}$ for each $n$, it must be nondecreasing on $\Gamma^{*}$. Since $\Gamma^{*}, U$, and $U_{x}$ are all $\tau$-periodic in $t$, we see that $-b U+a U_{x}$ is constant on $\Gamma^{*}$. Now $U=1$ on $\Gamma^{*}$ and so $U_{x}$ is constant on $\Gamma^{*}$. Thus the $\tau$-periodic function $g \equiv U_{x}$ is a solution of the boundary value problem

$$
\begin{aligned}
g_{t}=g_{x x} & & \text { in } \Omega \\
g=-1 & & \text { on } \Gamma \\
g=C & & \text { on } \Gamma^{*} .
\end{aligned}
$$

Then $U_{x}(x, t)=(C+1) U-1$ in $\bar{\Omega}$ and so $U_{t}=U_{x x}=(C+1) U_{x}$ in $\bar{\Omega}$. Thus $\Gamma$ and $\Gamma^{*}$ are straight lines and, since they are periodic in $t$, they are vertical. This contradiction implies that $t(s)$ does not approach $+\infty$. A similar argument shows that $t(s)$ does not approach $-\infty$. 
REMARK. It does not follow from the $\tau$-periodicity of $U, U_{x}, U_{t}$, etc. that maximal connected sets of the form $\gamma=\left\{-b U_{x}+a U_{t}=0\right\}$ are $\tau$-periodic. There are no $\tau$-periodic connected sets $\gamma=\left\{-b U_{x}+\right.$ $\left.a U_{t}=0\right\}$, since $-b U+a U_{x}$ would be monotonic and $\tau$-periodic on $\gamma$.

LEMma 16. Suppose the hypotheses of Lemma 15 are satisfied. Then $\gamma$ must terminate at a point $p_{0}^{*} \in \Gamma^{*}$ with the same normal vector (to $\left.\Gamma^{*}\right) \nu=a \vec{i}+b \vec{j}$.

Proof. Suppose $p_{0}=\left(x_{0}, t_{0}\right) \in \Gamma$ is a $\nu$-minimum and $\gamma$ begins at $p_{0}$. Let $(x(s), t(s))$ be an arclength parametrization of $\gamma$ and let $f=-b U+a U_{x}$. Now $\gamma$ remains in a bounded subset of $\Omega$. Further, once it leaves a neighborhood of $p_{0}, \gamma$ remains bounded away from $\Gamma$. Moreover, it can be shown using Lemmas 10, 11, and 14 that $\gamma$ has no accumulation points in $\Omega$. Since $\gamma$ does not end at a point of $\Omega$, there must be a terminal point or an accumulation point $p_{0}^{*}=\left(x_{0}^{*}, t_{0}^{*}\right) \in \Gamma^{*}$ of $\gamma$. Since $\nabla U$ is continuous on $\bar{\Omega}$ and $U_{\lambda}=0$ at each point of $\gamma, U_{\lambda}\left(p_{0}^{*}\right)=0$; thus the normal to $\Gamma^{*}$ at $p_{0}^{*}$ is $\nu=a \vec{i}+b \vec{j}$ (recall $\lambda=-b \vec{i}+a \vec{j}$ ). Since $t(s)$ is monotonic (at least for $\left.s \geq s_{0}\right), t(s) \rightarrow t_{0}^{*}$ as $s \rightarrow|\gamma|(|\gamma|$ may be $\infty)$. If $x(s)$ does not have a limit as $s \rightarrow \infty$, then there exists $\delta \rightarrow 0$ such that each point of $\left[x_{0}^{*}, x_{0}^{*}+\delta\right] \times\left\{t_{0}^{*}\right\}$ is an accumulation point of $\gamma$ and hence $U_{\lambda}=0$ on this interval, a horizontal line segment in $\Gamma^{*}$. This is not possible, since $\Gamma^{*}=\left\{(x, t): x=X^{*}(t)\right\}$, and so $x(s) \rightarrow x_{0}^{*}$ as $s \rightarrow|\gamma|$.

REMARK. Everything above carries over when $p_{0} \in \Gamma$ is a $\nu$-maximum except that $-b U+a U_{x}$ is decreasing on $\gamma$.

LemMa 17. Suppose $\Gamma^{*}$ is not a vertical line. Suppose $a>0, b \geq 0$, and $p_{0}=\left(x_{0}, t_{0}\right) \in \Gamma$ is a $\nu$-minimum of $\Gamma$, where $\nu=a \vec{i}+b \vec{j}$. Let $\gamma$ be the curve of constant gradient direction beginning at $p_{0}$ as in Lemma 16 and let $\gamma$ terminate at $p_{1}=\left(x_{1}, t_{1}\right) \in \Gamma^{*}$. Suppose that $x \geq x_{1}$ for every point $(x, t) \in \gamma$. Then $\nu \cdot\left(p_{0}-p_{1}\right)>a$.

Proof. Let us consider the path independent integral

$$
I=\int_{p_{0}}^{p_{1}}\left(-b U+a U_{x}\right) d x+\left(-b U_{x}+a U_{t}\right) d t
$$

If we integrate along $\gamma$ from $p_{0}$ to $p_{1}$ and integrate by parts, we see 
that

$$
I=\int_{p_{0}}^{p_{1}}\left(-b U+a U_{x}\right) d x
$$

and so

$$
I=\left.\left(x-x_{1}\right)\left(-b U+a U_{x}\right)\right|_{p_{0}} ^{p_{1}}-\int_{p_{0}}^{p_{1}}\left(x-x_{1}\right) d\left(-b U+a U_{x}\right) .
$$

Since $-b U+a U_{x}$ is increasing along $\gamma$ and $x-x_{1} \geq 0$, we see that $I<a\left(x_{0}-x_{1}\right)$.

On the other hand, $I=a \int_{p_{0}}^{p_{1}} U_{x} d x+U_{t} d t-b \int_{p_{0}}^{p_{1}} U d x+U_{x} d t$, and the first integral equals $a$. If we integrate the second integral first along $\Gamma$ from $p_{0}$ to $q_{0} \equiv\left(x_{3}, t_{1}\right)$ and then along the horizontal line from $q_{0}$ to $p_{1}$, we see that $I>a+b\left(t_{1}-t_{0}\right)$. If we combine the two inequalities for $I$, we obtain $\nu \cdot\left(p_{0}-p_{1}\right)>a$.

LEMMA 18. Suppose $\sigma$ is any curve from a point $p_{4}=\left(x_{4}, t_{4}\right) \in \Omega$ to a point $p_{1}=\left(x_{1}, t_{1}\right) \in \Gamma^{*}$ with $x_{4}>x_{1}$ along which $U_{t}=0$ and $U_{t}>0$ locally to the right of $\sigma$. Then $x>x_{1}$ for all points $(x, t) \in \sigma$.

Proof. Suppose first that the curve $\sigma$ stays to the right of the vertical line $x=x_{1}$ near $p_{1}$. Suppose $\sigma$ crosses the line $x=x_{1}$ at a point $p_{3}=\left(x_{1}, t_{3}\right)$ and stays to the right of the vertical line between $p_{3}$ and $p_{1}$. Let $p_{2}=\left(x_{2}, t_{2}\right) \in \sigma$ be the furthest point to the right on $\sigma$ between $p_{3}$ and $p_{1}$. From the monotonicity of $U-x$ on $\sigma$, we see that for some number $D>0, U_{x}<-D$ on $\sigma$ between $p_{3}$ and $p_{2}$ and $U_{x}>-D$ on $\sigma$ between $p_{2}$ and $p_{1}$. Then

$$
U\left(p_{3}\right)-U\left(p_{1}\right)=\int_{p_{1}}^{p_{3}} U_{x} d x+U_{t} d t=\int_{p_{1}}^{p_{3}} U_{x} d x>0 .
$$

Thus $U\left(p_{3}\right)>U\left(p_{1}\right)=1$ in violation of the maximum principle.

Suppose next that $\sigma$ lies to the left of the line $x=x_{1}$ near $p_{1}$. Let us assume that in a neighborhood of $p_{1}, \sigma$ stays above $t=t_{1}$ and between $\Gamma^{*}$ and $x=x_{1}$. Then for some $\varepsilon>0, U_{t}\left(X^{*}(t), t\right)<0$ for $t_{1}<t<t_{1}+\varepsilon$. Since $U_{t}>0$ to the right of $\sigma$, there must be a curve $\tilde{\sigma}$ which begins at $p_{1}$ and lies between $\Gamma^{*}$ and $\sigma$ along which $U_{t}=0$ and $U_{x}$ increases as points move away from $p_{1}$. This curve $\tilde{\sigma}$ must either terminate at a point $p_{5}$ of $\Gamma^{*}$ or cross the line $x=x_{1}$ at a point $p_{6}$. If we argue in a similar manner to the first paragraph, we obtain either $U\left(p_{5}\right)>U\left(p_{1}\right)$ or $U\left(p_{6}\right)>U\left(p_{1}\right)$; in either case, a contradiction results. 
Finally, if neither case holds, then $\sigma$ intersects the line $x=x_{1}$ infinitely often. An argument similar to the argument of the first paragraph implies that there is a sequence $\left(q_{n}\right)$ (of "every other point of intersection of $\sigma$ and $x=x_{1}$ ") such that $q_{n}$ converges to $p_{1}$ as $n \rightarrow \infty$ and $U\left(q_{n}\right)<U\left(q_{n+1}\right)$ for each $n$. Since $U$ is continuous, this contradicts the maximum principle.

REMARK. Using a similar (but more complicated) proof, it can be shown that a curve $\sigma$ as above with $U_{\lambda}=0$ on $\sigma$ and $U_{\lambda}>0$ locally to the right of $\sigma$ cannot cross the tangent line $a\left(x-x_{1}\right)+b\left(t-t_{1}\right)=0$.

THEOREM 4. (a) If $\Gamma$ has $\nu$-minima ( $\nu$-maxima) in one period (i.e. in $\left.\Gamma \cap\left(\Re \times\left[t_{0}, t_{0}+\tau\right]\right)\right)$, then $\Gamma^{*}$ has at least $n \nu$-minima ( $\nu$-maxima) in one period.

(b) The total curvature of $\Gamma$ in a single period cannot exceed the total curvature of $\Gamma^{*}$ in one period.

(c) The $x$-variation of $\Gamma$ in one period (i.e. $\int_{0}^{\tau}\left|x^{\prime}(t)\right| d t$, where $(X(t), t)$ is a parametrization of $\Gamma)$ cannot exceed that of $\Gamma^{*}$ in one period.

Proof. Suppose $p_{k}^{0}=\left(x_{k}^{0}, t_{k}^{0}\right) \in \Gamma, k=1, \ldots, n$, with $t_{1}^{0} \leq t_{2}^{0} \leq$ $\cdots \leq t_{n}^{0}<t_{1}^{0}+\tau$ such that $p_{k}^{0}$ is a $\nu$-minimum of $\Gamma, k=1, \ldots, n$, and, for some $t \in\left(t_{k}, t_{k+1}\right), U_{\lambda}(X(t), t) \neq 0$, for each $1 \leq k \leq n-1$. For each $k=1, \ldots, n$, there is a curve $\gamma_{k}$ starting at $p_{k}^{0}$ and ending at a point $p_{k}^{*} \in \Gamma^{*}$ such that $U_{\lambda}=0$ on $\gamma_{k}$ and $U_{\lambda}>0$ locally to the right of $\gamma_{k}$. Then $\nu$ is a normal to $\Gamma^{*}$ at $p_{k}^{*}, k=1, \ldots, n$, and the curves $\gamma_{k}$ do not intersect in $\bar{\Omega}$. To see this, let $q \in \Gamma$ lie between $p_{k}$ and $p_{k+1}$ such that $q$ is a $\nu^{*}$-minimum of $\Gamma$, where $\nu^{*}$ is not parallel to $\nu$. Let $\sigma_{1}$ be the curve of constant gradient direction beginning at $q$ and ending at a point $q_{1} \in \Gamma^{*}$. If $\sigma_{1}$ intersects $\gamma_{k}$, for example, at $p$, then $\nabla U(p)=\overrightarrow{0}$. From Lemma 2 and the maximum principle, we see that $U_{x} \neq 0$ in $\bar{\Omega}$ and so $\sigma_{1} \cap \gamma_{k}=\varnothing$. Since $\sigma_{1}$ separates $\gamma_{k}$ and $\gamma_{k+1}$ near $\Gamma$, the $\gamma_{k}$ cannot intersect in $\bar{\Omega}$. Notice then that $p_{k}^{*} \neq p_{k+1}^{*}, 1 \leq k \leq n-1$.

Next, let us fix $k \in\{1, \ldots, n\}$. Let $q_{1} \in \Gamma$ with $q_{1}$ between $p_{k}$ and $p_{k+1}$ and $q_{2} \in \Gamma$ with $q_{2}$ between $p_{k-1}$ and $p_{k}$ such that $\nu_{1}=$ $a \vec{i}+b_{1} \vec{j}$ and $\nu_{2}=a \vec{i}+b_{2} \vec{j}$ are (exterior) normals to $\Gamma$ at $q_{1}$ and $q_{2}$ respectively with $b_{1}<b<b_{2}$ and $q_{1}$ and $q_{2}$ are strict local minima of $\Gamma$ with respect to their normals $\nu_{k}$ (where $p_{n+1} \equiv p_{1}+$ $(0, \tau)$ and $\left.p_{0} \equiv p_{n}-(0, \tau)\right)$. Let $\tilde{\gamma}_{1}$ and $\tilde{\gamma}_{2}$ be curves of constant gradient direction beginning at $q_{1}$ and $q_{2}$ respectively. Then $\tilde{\gamma}_{1}$ and 
$\tilde{\gamma}_{2}$ terminate at points $q_{1}^{*}$ and $q_{2}^{*}$ of $\Gamma^{*}$ respectively and $\nu_{1}$ and $\nu_{2}$ are (interior) normals to $\Gamma^{*}$ at $q_{1}^{*}$ and $q_{2}^{*}$ respectively. Since $\Gamma^{*}$ is $C^{1}$ and $p_{k}^{*}$ lies between $q_{1}^{*}$ and $q_{2}^{*}$, there must be a point $p_{k}^{* *} \in \Gamma^{*}$ at which $\Gamma^{*}$ has a $\nu$-minimum (in the sense mentioned at the beginning of this section). The proof of (a) and (b) follows.

Suppose $p_{0}=\left(x_{0}, t_{0}\right) \in \Gamma$ is a $(1,0)$-minimum of $\Gamma$ and $p_{2}=$ $\left(x_{2}, t_{2}\right) \in \Gamma$ is the next $(1,0)$-maximum of $\Gamma$. Suppose that $\gamma$ and $\tilde{\gamma}$ are curves of constant gradient direction starting from $p_{0}$ and $p_{2}$ respectively as in Lemma 16 and let these curves terminate at points $p_{1}=\left(x_{1}, t_{1}\right)$ and $p_{3}=\left(x_{3}, t_{3}\right)$ of $\Gamma^{*}$ respectively. Notice that $U_{t}>0$ locally to the left of $\tilde{\gamma}$. Let $a=1$ and $b=0$. From Lemmas 17 and 18 , we see that $x_{0}-x_{1}>1$. If we apply the first part of the proof of Lemma 17 to $\tilde{\gamma}$, we see that $I>x_{2}-x_{3}$. (An argument similar to that of Lemma 18 shows that $\tilde{\gamma}$ cannot cross the line $x=x_{3}$.) Since $I=a=1$, we obtain $x_{2}-x_{3}<1$. Thus the $x$-variation of $\Gamma$ between $p_{0}$ and $p_{2}$ is less than the $x$-variation of $\Gamma^{*}$ between $p_{1}$ and $p_{3}$. The last part follows from this.

REMARK. Results similar, for example, to Theorem 4 of [4] and Theorem 5 of [6] for this problem follow from our methods.

REMARK. The results in $\S \S 2$ and 3 were obtained by the first author (Acker) while he was a guest of SFB 123, University of Heidelberg, in summer, 1987. Section 4 represents joint work.

\section{REFERENCES}

[1] A. Acker, Free boundary optimization-a constructive iterated method, J. Appl. Math. Phys. (ZAMP), 30 (1979), 885-900.

[2] - How to approximate the solutions of certain free-boundary problems for the Laplace equation by using the contraction principle, J. Appl. Math. Phys. (ZAMP), 32 (1981), 22-33.

[3] _ On the geometric form of free boundaries satisfying a Bernoulli condition, Math. Methods Appl. Sci., 6 (1984), 449-456.

[4] On the geometric form of free boundaries satisfying a Bernoulli condition, II, Math. Methods Appl. Sci., (1986), 387-404.

[5] Convergence results for an analytical trial free-boundary method, IMA J. Numer. Anal., 8 (1988), 357-364.

[6] A. Acker and K. Lancaster, The geometry of curves of constant contact angle for doubly-connected minimal surfaces, Comm. Partial Differential Equations, 14 (1989), 375-390.

[7] G. Alessandrini, An identification problem for an elliptic equation in two variables, (manuscript).

[8] S. Angenent, The zero set of a solution of a parabolic equation, J. Reine Angew. Math., 390 (1988), 79-96. 
[9] C. Baiocchi and A. Capelo, Variational and Quasivariational Inequalities, Wiley, Chichester, 1984.

[10] G. Birkhoff and E. Zarantonello, Jets, Wakes, and Cavities, Academic Press, New York, 1957.

[11] J. Cannon, The One-Dimensional Heat Equation, Addison-Wesley, Menlo Park, 1984.

[12] M. Chipot, Variational Inequalities and Flow in Porous Media, Springer, New York, 1984.

[13] R. Courant and D. Hilbert, Methods of Mathematical Physics, vol. 1, Springer, New York, 1970.

[14] J. Crank, Free and Moving Boundary Problems, Clarendon Press, Oxford, 1984.

[15] A. Crowley and C. Elliott (editors). Proceedings of the Conference on Crystal Growth, IMA J. Appl. Math., (1985), 115-264.

[16] C. Cryer, Numerical Methods for Free and Moving Boundary Problems, (presented at State of the Art in Numerical Analysis, Birmingham, April, 1986).

[17] R. Finn, Equilibrium Capillary Surfaces, Springer, New York, 1986.

[18] A. Friedman, Variational Principles and Free Boundary Problems, Wiley, New York, 1982.

[19] A. Friedman and R. Jensen, Convexity of the free boundary in the Stefan problem and in the dam problem, Arch. Rational Mech. Anal., 67 (1978), 1-24.

[20] A. Friedman and T. Vogel, Cavitational flow in a channel with oscillatory wall, Nonlinear Analysis TMA, 7 (1983), 1175-1192.

[21] E. Holmgren, Sur une application de l'equation integrale de M. Volterra, Ark. für Mathematik, Astronomi och Fysik, 3 (1907), 1-4.

[22] D. Kinderlehrer and Nirenberg, Regularity in free boundary problems, Ann. Scuola Norm. Sup. Pisa, ser. IV, 4 (1977), 373-391.

[23] M. Lavrentiev, Variational Methods, Groningen, 1963.

[24] M. Lavrentiev and B. Schabat, Methoden der komplexen Funktionentheorie, Berlin, 1967.

[25] K. Nickel, Einige Eigenschaften von Lösungen der Pradtlschen GrenzschichtDifferentialgleichungen, Arch. Rational Mech. Anal., 2 (1958), 1-31.

[26] __ Gestaltaussagen uber Losungen parabolischer Differentialgleichungen, J. Reine Angew. Math., 211 (1962), 78-94.

[27] P. Panagiotopoulos, Inequality Problems in Mechanics and Applications, Birkhäuser, Boston, 1985.

[28] L. Payne, On two conjectures in the fixed membrane eigenvalue problem, J. Appl. Math. Phys. (ZAMP), 24 (1973), 721-729.

[29] M. Protter and H. Weinberger, Maximum Principles in Partial Differential Equations. Springer-Verlag, 1970.

[30] L. Rubinštein, The Stefan Problem, Transl. Math. Monographs, vol. 27, Amer. Math. Soc., Providence, R.I., 1971.

[31] A. Schatz, Free boundary problems of Stephen type with prescribed flux, J. Math. Anal. Appl., 28 (1969), 569-580.

[32] W. Walter, Differential and Integral Inequalities, Springer-Verlag, New York, 1970 .

Received October 12, 1988.

The Wichita State University

WichITA, KS 67208 


\section{PACIFIC JOURNAL OF MATHEMATICS EDITORS}

\author{
V. S. VARADARAJAN \\ (Managing Editor) \\ University of California \\ Los Angeles, CA 90024-1555-05 \\ Herbert Clemens \\ University of Utah \\ Salt Lake City, UT 84112 \\ THOMAS ENRIGHT \\ University of California, San Diego \\ La Jolla, CA 92093
}

R. FINN

Stanford University

Stanford, CA 94305

HeRmann FlaschKa

University of Arizona

Tucson, AZ 85721

VAUghan F. R. Jones

University of California

Berkeley, CA 94720

SteVEN KeRCKHOFF

Stanford University

Stanford, CA 94305

\section{C. MOORE}

University of California

Berkeley, CA 94720

MARTIN SCHARLEMANN

University of California

Santa Barbara, CA 93106

HAROLD STARK

University of California, San Diego

La Jolla, CA 92093

\section{ASSOCIATE EDITORS \\ R. ARENS \\ E. F. BECKENBACH \\ B. H. NeUmanN \\ F. WoLF \\ (1904-1989) \\ K. Yoshida (1906-1982)}

\section{SUPPORTING INSTITUTIONS}

UNIVERSITY OF ARIZONA

UNIVERSITY OF BRITISH COLUMBIA

CALIFORNIA INSTITUTE OF TECHNOLOGY

UNIVERSITY OF CALIFORNIA

MONTANA STATE UNIVERSITY

UNIVERSITY OF NEVADA, RENO

NEW MEXICO STATE UNIVERSITY

OREGON STATE UNIVERSITY
UNIVERSITY OF OREGON

UNIVERSITY OF SOUTHERN CALIFORNIA

STANFORD UNIVERSITY

UNIVERSITY OF HAWAII

UNIVERSITY OF TOKYO

UNIVERSITY OF UTAH

WASHINGTON STATE UNIVERSITY

UNIVERSITY OF WASHINGTON

The Supporting Institutions listed above contribute to the cost of publication of this Journal, but they are not owners or publishers and have no responsibility for its content or policies.

Mathematical papers intended for publication in the Pacific Journal of Mathematics should be in typed form or offset-reproduced (not dittoed), double spaced with large margins. Please do not use built up fractions in the text of the manuscript. However, you may use them in the displayed equations. Underline Greek letters in red, German in green, and script in blue. The first paragraph must be capable of being used separately as a synopsis of the entire paper. In particular it should contain no bibliographic references. Please propose a heading for the odd numbered pages of less than 35 characters. Manuscripts, in triplicate, may be sent to any one of the editors. Please classify according to the 1991 Mathematics Subject Classification scheme which can be found in the December index volumes of Mathematical Reviews. Supply name and address of author to whom proofs should be sent. All other communications should be addressed to the managing editor, or Elaine Barth, University of California, Los Angeles, California 90024-1555-05.

There are page-charges associated with articles appearing in the Pacific Journal of Mathematics. These charges are expected to be paid by the author's University, Government Agency or Company. If the author or authors do not have access to such Institutional support these charges are waived. Single authors will receive 50 free reprints; joint authors will receive a total of 100 free reprints. Additional copies may be obtained at cost in multiples of 50 .

The Pacific Journal of Mathematics (ISSN 0030-8730) is published monthly except for July and August. Regular subscription rate: $\$ 190.00$ a year (10 issues). Special rate: $\$ 95.00$ a year to individual members of supporting institutions.

Subscriptions, orders for numbers issued in the last three calendar years, and changes of address should be sent to Pacific Journal of Mathematics, P.O. Box 969, Carmel Valley, CA 93924, U.S.A. Old back numbers obtainable from Kraus Periodicals Co., Route 100, Millwood, NY 10546.

The Pacific Journal of Mathematics at P.O. Box 969, Carmel Valley, CA 93924 (ISSN 0030-8730) is published monthly except for July and August. Second-class postage paid at Carmel Valley, California 93924, and additional mailing offices. Postmaster: send address changes to Pacific Journal of Mathématics, P.O. Box 969, Carmel Valley, CA 93924.

PUBLISHED BY PACIFIC JOURNAL OF MATHEMATICS, A NON-PROFIT CORPORATION Copyright (C) 1991 by Pacific Journal of Mathematics 


\section{Pacific Journal of Mathematics}

Vol. 148, No. $2 \quad$ April, 1991

Yuri A. Abramovich, Operators preserving disjointness on rearrangement

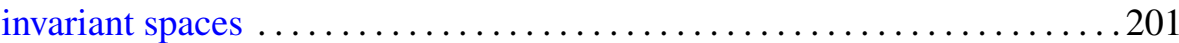

Andrew French Acker and Kirk Lancaster, Existence and geometry of a free boundary problem for the heat equation .................. 207

So-Chin Chen, Real analytic regularity of the Szegő projection on circular

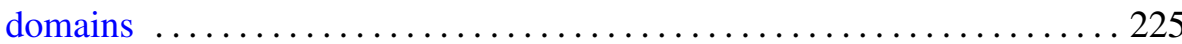

Chen-Lian Chuang, An independence property of central polynomials . . . 237

Peter Larkin Duren and M. Schiffer, Robin functions and energy

functionals of multiply connected domains $\ldots \ldots \ldots \ldots \ldots \ldots \ldots \ldots . \ldots 251$

Johan Henricus Bernardus Kemperman, Sets of uniqueness and systems

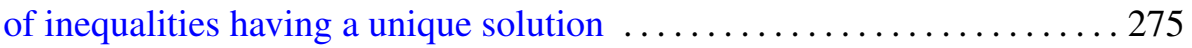

Ka-Lam Kueh, Fourier coefficients of nonholomorphic modular forms and sums of Kloosterman sums . .......................... 303

Gerard J. Murphy, Ordered groups and crossed products of $C^{*}$-algebras . . 319

You-Qiang Wang, The $p$-parts of Brauer character degrees in $p$-solvable

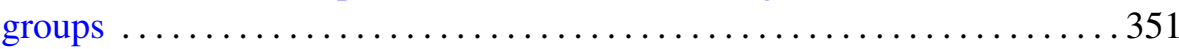

Hidenobu Yoshida, Harmonic majorization of a subharmonic function on a cone or on a cylinder ........................................ 369 